\title{
Application of primed in situ DNA synthesis (PRINS) with telomere human commercial kit in molecular cytogenetics of Equus caballus and Sus scrofa scrofa
}

\author{
Maciej Wnuk ${ }^{1}$, Monika Bugno², Ewa Slota ${ }^{2}$ \\ ${ }^{1}$ Department of Genetics, University of Rzeszów, Rzeszów, Poland \\ ${ }^{2}$ Department of Immuno- and Cytogenetics, National Research Institute of Animal Production, \\ Balice n. Kraków, Poland
}

\begin{abstract}
Recently, molecular techniques have become an indispensable tools for cytogenetic research. Especially, development of in situ techniques made possible detection at the chromosomal level, genes as well as repetitive sequences like telomeres or the DNA component of telomeres. One of these methods is primed in situ DNA synthesis (PRINS) using an oligonucleotide primer complementary to the specific DNA sequence. In this report we described application of PRINS technique with telomere human commercial kit to telomere sequences identification. This commercial kit may be use to visualization of interstitial telomeric signal in pig genome. PRINS is attractive complement to FISH for detection of DNA repetitive sequences and displays lower level of non-specific hybridization than conventional FISH.
\end{abstract}

Key words: PRINS - Telomere - Pig - Horse

\section{Introduction}

Development of the molecular cytogenetic techniques results in utilization of the fluorescence in situ hybrizdiation (FISH) technique in cytogenetic research [20].

Alternative method of chromosome investigations is primed in situ DNA synthesis (PRINS) technique. In this method unlabeled oligonucleotides that are annealed in situ to complementary sequences in metaphase chromosomes or interphase nuclei and extended with Taq polymerase. The major advantages of PRINS reaction are rapidity and the high sensitivity as the PCR which makes possible the chromosomal localization of specific DNA sequences [10,29].

PRINS can be applied especially in detection of repetitive sequences like: alphoid sequences, satellite sequences, Alu-dispersed sequences, rDNA sequences, heterochromatin or telomeres $[6,10,14,16,34]$.

PRINS technique can be used as a gene mapping technique, anueploidy diagnostic method as well as in

Correspondence: M. Wnuk, Department of Genetics, University of Rzeszów, Rejtana Str. 16C, 35-959 Rzeszów, Poland; tel.: (+4817) 8723704, fax.: (+4817) 8723708,

e-mail:mawnuk@gmail.com studies concerning evolutional analysis or evaluation of DNA damage [6,12,29-31].

Moreover PRINS can be applied in flow cytometry and sorting chromosomes (flow cytogenetics) $[8,9,18,22,23]$.

In this paper, we have shown that PRINS technique with telomere human commercial kit may be applied in cytogenetic research of economically important group of mammals.

\section{Material and methods}

Chromosome preparation. Metaphase spreads of pig and horse chromosomes were obtained after routine lymphocytes culture. Preparations were aged for at least 1 week at $37^{\circ} \mathrm{C}$.

Oligonucleotide - primed in situ DNA synthesis. We applied the standard protocol according to the manufacture instruction with minor modifications. Metaphase spreads were treated with RNase $\left(1 \mathrm{~h}\right.$ at $37^{\circ} \mathrm{C}$ in humid chamber ) and next with pepsin solution (at $37^{\circ} \mathrm{C}$ for $30 \mathrm{~min}$ ) for better results. After pepsin digestion slides were washed twice in PBS (pH 7.4) and passed through the ethanol series $(70,80$ and $95 \%)$. Before air-drying, the reaction mix was warmed to $37^{\circ} \mathrm{C}$ for $5 \mathrm{~min}$ and mixed. $25 \mu \mathrm{l}$ of reaction mixture containing PRINS Kit (Prins-Probe 1409-T, Cambio Ltd) $0.05 \mathrm{mM}$ biotin-11-dUTP and 2 U Taq polymerase (Eppendorf) was added to air-dried slide. Reaction mixture was put on the $20 \times 40 \mathrm{~mm}^{2} \mathrm{cov}-$ erslip, was placed on the slide and quickly sealed with fixogum. The slide was placed on preheated block at $94^{\circ} \mathrm{C}$ for $5 \mathrm{~min}$ and next 

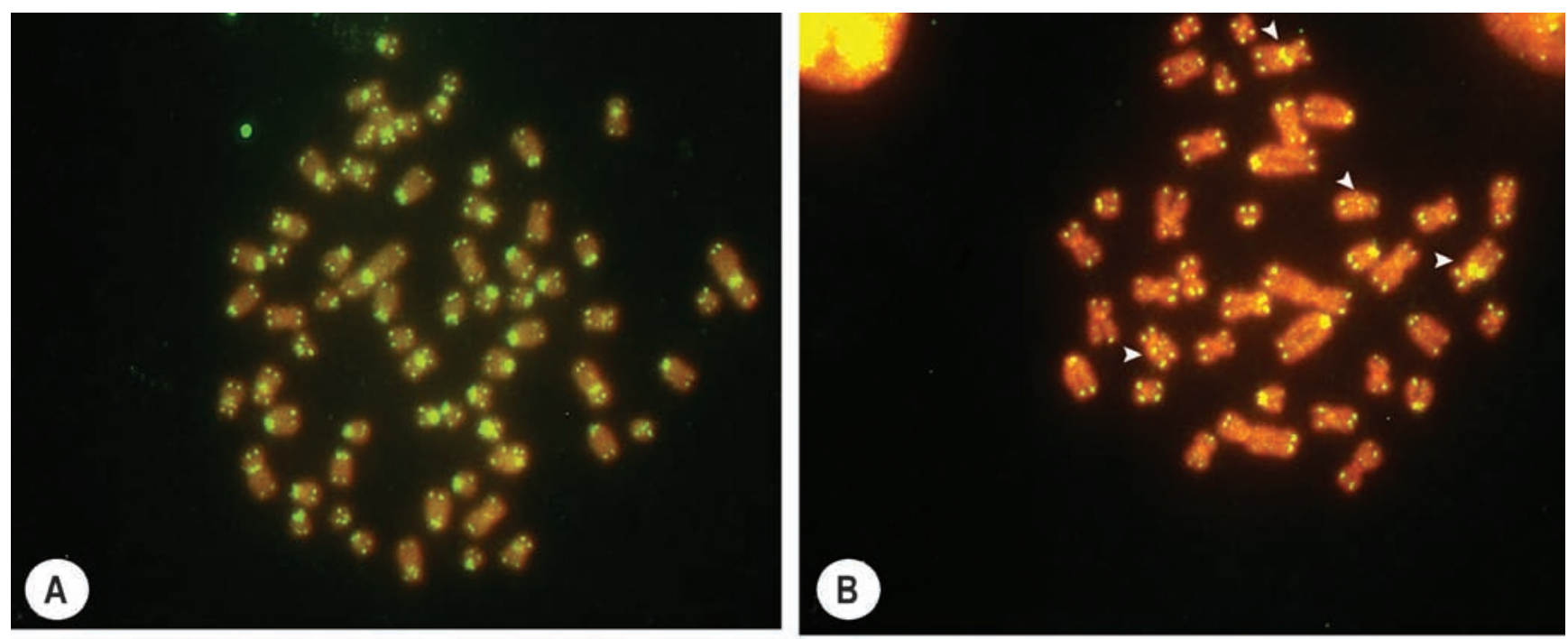

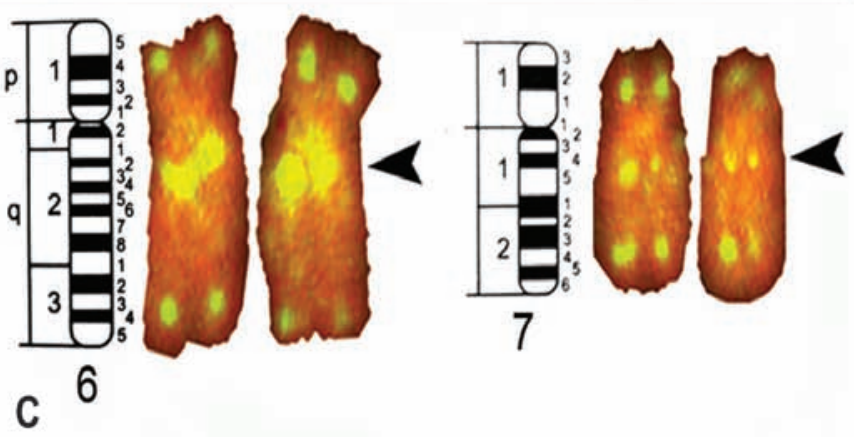

Fig 1. Two domestic horse (A) and domestic pig (B) metaphase spreads after PRINS with telomere human commercial kit. (A) Horse metaphase spread shows fluorescence signals in centromeres as well as on the ends of all chromosomes. (B) Pig metaphase spread shows fluorescence signals on the ends of the all chromosomes and in centromers of all acrocentric chromosomes. Arrowhead indicate additional intrachromosomal signal on $6 \mathrm{q}$ and 7q chromosomes. (C) Chromosomes of 6 and 7 pairs and idiograms. Arrowheads indicate additional intrachromosomal signal on $\mathrm{q}$ arm of both homologous of 6 and 7 chromosomes.

The telomeric repeats can be detected either by using fluorescence in situ hybridization (FISH) technique with a DNA probe or by primed in situ labeling (PRINS) reaction using an oligonucleotide primer complementary to the telomeric DNA repeated sequence.

In this examination, application of primed in situ DNA synthesis (PRINS) method with telomeric oligonucleotide in pig and horse metaphase spreads gave fluorescence signals on both ends of all chromosomes (Fig. 1A and 1B). These results are consistent with previous study carried out on both species using FISH with telomeric DNA probe $[7,19]$. However, our results proved that PRINS technique is fast, attractive and alternative method than conventional FISH for in situ detection of telomeric sequences [7].

Moreover, the telomere - specific oligonucleotide revealed centromeric fluorescence signals on all horse chromosomes (Fig. 1A). This finding indicated that it is some similarity between both repetitive sequences.

In pig, PRINS method did not give any signals in centromers of metacentric chromosomes of pairs 1 to 12 as well as X (Fig. 1B). In parallel, PRINS revealed fluorescence signals in all acrocentric chromosomes (pairs 13 to 18). These findings confirm that pigs have two distinct centromeric DNA satellite families, Mc1 and Ac2 [15]. The centromeres of Mc1 family are 
comprised of divergent $\sim 340 \mathrm{bp}$ monomer units $[15,27,33]$. The centromeres of Ac2 family are composed of $14 \mathrm{bp}$ monomer units [32].

Additionally, we showed strong interstitial telomeric signal (ITS) on both homologues of chromosome pair 6q (Fig. 1B and 1C). This data confirm previous observation using FISH with telomeric sequences [7]. Also, we observed another class of telomeric fluorescence signal on 7q chromosomes (Fig. 1B and 1C). However, both signals on $7 \mathrm{q}$ chromosomes were weaker than on both homologues of chromosome pair $6 \mathrm{q}$ and they could be related to interstitial CG-rich sequences [2].

The intrachromosomal telomeric repeats have been described in a variety of vertebrates ranging from mammals [24,35], birds [28], amphibian [36] to fishes [1]. The (TTAGGG)n sequence located between centromere and telomere can be the result of tandem chromosome fusion during evolution as well as the insertion of telomereic DNA within unstable sites during the repair of interstitial double-strand breaks $[3,6,11,13]$. Moreover, these sequences can be detected in many species at various nontelomeric sites, predominantly in pericentromeric regions and other regions rich in constitutive heterochromatin $[25,35]$. The heterochromatic nature of mammalian telomeres is responsible for transcriptional silencing of nearby genes as well as the suppression of recombination [17].

In this report, we demonstrated the application of PRINS technique with telomere human commercial kit to detect the telomeres and centromeres in horse karyotype. Additionally, the same PRINS kit can be used to labeling both the telomeric, interstitial telomeric signals (ITS) and centromers of acrocentric chromosomes in pig karyotype.

\section{References}

[1] Abuin M, Martinez P, Sanchez L. Localization of the repetitive telomeric sequence (TTAGGG)n in four salmonid species. Genome. 1996;39:1035-1038.

[2] Adega F, Chaves R, Guedes-Pinto H. Chromosome restriction enzyme digestion in domestic pig (Sus scrofa) constitutive heterochromatin arrangement. Genes Genet Syst. 2005; 80:49-56.

[3] Azzalin CM, Nergadze SG, Giulotto E. Human intrachromosomal telomeric-like repeats: sequence organization and mechanisms of origin. Chromosoma. 2001;110:75-82.

[ 4] Biessmann H, Mason JM. Telomeric repeat sequences. Chromosoma. 1994; 103:154-161.

[ 5] Blackburn EH. Telomeres and telomerase. Keio J Med. 2000; 49:59-65.

[6] Bolzan AD, Bianchi MS. Telomeres, interstitial telomeric repeat sequences, and chromosomal aberrations. Mutat Res. 2006;612:189-214.

[7] de la Sena C, Chowdhary BP, Gustavsson I. Localization of the telomeric (TTAGGG)n sequences in chromosomes of some domestic animals by fluorescence in situ hybridization. Hereditas. 1995;123:269-274.
[ 8] Dolezel J, Kubalakova M, Bartos J, Macas J. Flow cytogenetics and plant genome mapping. Chromosome Res. 2004; 12:77-91.

[9] Fischer H, Hindkjaer J, Pedersen S, Koch J, Brandt C, Kolvraa S. Primed in situ labeling (PRINS) and fluorescence in situ hybridization (FISH). Methods Cell Biol. 1994;42 Pt B:71-93.

[10] Gosden J, Hanratty D, Starling J, Fantes J, Mitchell A, Porteous D. Oligonucleotide-primed in situ DNA synthesis (PRINS): a method for chromosome mapping, banding, and investigation of sequence organization. Cytogenet Cell Genet. 1991;57:100-104.

[11] Hastie ND, Allshire RC. Human telomeres: fusion and interstitial sites. Trends Genet. 1989;5:326-331.

[12] Hirai H. Relationship of telomere sequence and constitutive heterochromatin in the human and apes as detected by PRINS. Methods Cell Sci. 2001;23:29-35.

[13] Holmquist GP, Dancis B. Telomere replication, kinetochore organizers, and satellite DNA evolution. Proc Natl Acad Sci USA. 1979;76:4566-4570.

[14] Jankun M, Ocalewicz K, Pardo BG, Martinez P, Woznicki P, Sanchez L. Localization of 5S rRNA loci in three coregonid species (Salmonidae). Genetica. 2003;119:183-186.

[15] Jantsch M, Hamilton B, Mayr B, Schweizer D. Meiotic chromosome behaviour reflects levels of sequence divergence in Sus scrofa domestica satellite DNA. Chromosoma. 1990;99: 330-335.

[16] Koch J. Detection and sizing of telomeric and other simple repeats by dideoxy-PRINS. Methods Mol Biol. 2006;334:8188 .

[17] Koering CE, Pollice A, Zibella MP, et al. Human telomeric position effect is determined by chromosomal context and telomeric chromatin integrity. EMBO Rep. 2002;3:1055-1061.

[18] Kubalakova M, Vrana J, Cihalikova J, Lysak MA, Dolezel J. Localisation of DNA sequences on plant chromosomes using PRINS and C-PRINS. Methods Cell Sci. 2001;23:71-82.

[19] Lear TL. Chromosomal distribution of the telomere sequence (TTAGGG)(n) in the Equidae. Cytogenet Cell Genet. 2001;93:127-130.

[20] Levsky JM, Singer RH. Fluorescence in situ hybridization: past, present and future. J Cell Sci. 2003;116:2833-2838.

[21] Lundblad V. DNA ends: maintenance of chromosome termini versus repair of double strand breaks. Mutat Res. 2000;451: 227-240.

[22] Lysak MA, Cihalikova J, Kubalakova M, Simkova H, Kunzel G, Dolezel J. Flow karyotyping and sorting of mitotic chromosomes of barley (Hordeum vulgare L.). Chromosome Res. 1999;7:431-444.

[23] Macas J, Navratilova A, Kubalakova M, Dolezel J. PRINS on plant chromosomes. Methods Mol Biol. 2006;334:133-139.

[24] Meyne J, Baker RJ, Hobart HH, et al. Distribution of nontelomeric sites of the (TTAGGG)n telomeric sequence in vertebrate chromosomes. Chromosoma. 1990;99:3-10.

[25] Meyne J, Moyzis RK. in situ hybridization using synthetic oligomers as probes for centromere and telomere repeats. Methods Mol Biol. 1994;33:63-74.

[26] Meyne J, Ratliff RL, Moyzis RK. Conservation of the human telomere sequence (TTAGGG)n among vertebrates. Proc Natl Acad Sci USA. 1989;86:7049-7053.

[27] Miller JR, Hindkjaer J, Thomsen PD. A chromosomal basis for the differential organization of a porcine centromere-specific repeat. Cytogenet Cell Genet. 1993;62:37-41.

[28] Nanda I, Schrama D, Feichtinger W, Haaf T, Schartl M, Schmid M. Distribution of telomeric (TTAGGG)(n) sequences in avian chromosomes. Chromosoma. 2002;111:215-227.

[29] Pellestor F. Development and adaptation of the PRINS technology: an overview. Methods Mol Biol. 2006;334:211-220. 
[30] Pellestor F, Andreo B, Puechberty J, Lefort G, Sarda P. Analysis of sperm aneuploidy by PRINS. Methods Mol Biol. 2006;334:49-59.

[31] Pellestor F, Quenesson I, Coignet L, et al. FISH and PRINS, a strategy for rapid chromosome screening: application to the assessment of aneuploidy in human sperm. Cytogenet Cell Genet. 1996;72:34-36.

[32] Riquet J, Mulsant P, Yerle M, et al. Sequence analysis and genetic mapping of porcine chromosome 11 centromeric S0048 marker. Cytogenet Cell Genet. 1996;74:127-132.

[33] Rogel-Gaillard C, Bourgeaux N, Save JC, et al. Construction of a swine YAC library allowing an efficient recovery of unique and centromeric repeated sequences. Mamm Genome. 1997;8:186-192.
[34] Russo A. PRINS tandem labeling of satellite DNA in the study of chromosome damage. Am J Med Genet. 2002;107: 99-104.

[35] Santani A, Raudsepp T, Chowdhary BP. Interstitial telomeric sites and NORs in Hartmann's zebra (Equus zebra hartmannae) chromosomes. Chromosome Res. 2002;10:527-534.

[36] Wiley JE, Meyne J, Little ML, Stout JC. Interstitial hybridization sites of the (TTAGGG)n telomeric sequence on the chromosomes of some North American hylid frogs. Cytogenet Cell Genet. 1992;61:55-57.

Submitted: 10 September, 2007 Accepted after reviews: 14 December, 2007 